\title{
A Cluster Based Job Scheduling Algorithm for Grid Computing
}

\author{
Reza Fotohi \\ Department of Computer Engineering, Germi branch, Islamic Azad University, Germi, Iran \\ E-mail:Fotohi.Reza@gmail.com \\ Mehdi Effatparvar \\ ECE Department, Ardabil Branch, Is lamic Azad University, Ardabil, Iran \\ E-mail:Me.Effatparvar@gmail.com
}

\begin{abstract}
Grid computing enables sharing, selection and aggregation of computing resources for solving complex and large-scale scientific problems. The resources making up a grid need to be managed to provide a good quality of service. Grid scheduling is a vital component of a Computational Grid infrastructure.

This paper presents a dynamic cluster based job scheduling algorith $\mathrm{m}$ for efficient execution of user jobs This paper also includes the comparative performance analys is of our proposed job scheduling algorithm along with other well-known job scheduling algorithms considering the parameters like average waiting time, average turnaround time, average response time and average total completion time.

The result has shown also exhibit that Our proposed scheduling algorithms ( $\left.\mathrm{CHS}^{1}\right)$ has shown the best average waiting times, average turnaround times, average response times and average total completion times compared to other job scheduling approaches.
\end{abstract}

Index Terms - Grid Computing, Scheduling Algorithm, Cluster based Hybrid Scheduling, CHS

\section{Introduction}

Computational grid has the potential for solving large-scale scientific problems using geographically distributed and heterogeneous resources. Grid scheduling is a vital component of a computational grid infrastructure, which plays an important role in the efficient and effective execution of various kinds of scientific and engineering applications [1, 2]. A grid system is formed using many heterogeneous or homogeneous resources to deal with large-scale scientific problems. There are many issues in using grid computing. How to appropriately and efficiently assign resources to tasks, generally called job scheduling, is one of the important issues. The main purpose of job scheduling is to shorten the job completion time and

${ }^{1}$ Cluster based Hybrid Scheduling (CHS) enhance the system throughput. A grid scheduling system should take the various characteristics of grid applications and resources into account. In a grid environment, the resource providers and tasks are all changing constantly, so the traditional scheduling algorithms, e.g. "First Come, First Serve" may not be suitable for a dynamic grid system. It is very important to assign appropriate resources to tasks. Through a good scheduling method, the system can perform better and applications can avoid unnecessary delays.

When science and technology advance, the problems encountered become more complicated and need more computing power. In contrast to the traditional notion of using supercomputers, grid computing is proposed. Distributed computing supports resource sharing. Parallel computing supports computing power. Grid computing aims to harness the power of both distributed computing and parallel computing. The goal of grid computing is to aggregate idle resources on the Internet such as Central Processing Unit (CPU) cycles and storage spaces to facilitate utilization. The Search for Extra-Terrestrial Intelligence (SETI) experiment [3] is an early application of grids. The data Trans-Atlantic Grid project (TAG) [4] constructs a large-scale intercontinental grid test bed which focuses on issues of advanced networking and interoperability between these intercontinental grid domains, hence extending the capabilities of each and enhancing the worldwide program of grid development.

In implementation, Globus Toolkit [5] is an open source and a fundamental enabling technology for grid. The latest version of Globus Toolkit is Globus Toolkit 5.2.0. Grid can achieve the same level of computing power as a supercomputer does, but at a much reduced cost. Grid is like a virtual supercomputer. However, we need to consider about many conditions such as network status and resource status because the members of grid are connected by networks. Grid is also a heterogeneous system. Scheduling independent tasks on it is more complicated. In order to utilize the power of grid computing completely, we need an efficient job scheduling algorithm to assign jobs to resources. This 
paper focuses on the efficient job scheduling considering the average waiting time, average turnaround time, average response time and average total completion time of jobs in a grid computing.

Grid scheduling presents several challenges that make the implementation of practical systems a very difficult problem. Our research aims to design and develop Grid scheduling algorithms that makes efficient utilization of resources, maintain a high level of performance and possess a high degree of scalability.

This paper presents a dynamic cluster based job scheduling algorith $m$ for efficient execution of user jobs. This paper also includes the comparative performance analys is of our proposed job scheduling algorithm along with other well-known job scheduling algorith ms e.g.; First Come First Served (FCFS), Longest Job First (LJF), Shortest Process Next (SPN), Round Robin (RR), Proportional Local Round Robin (PLRR), Multilevel Dual Queue (MDQ). We evaluated the performance and scalability of each scheduling algorithm on a computational grid using six key performance parameters, i.e. average waiting time, average turnaround time, average response time and average total completion time in a grid computing.

The remainder of this paper is organized as follows: Section 2 gives related research. Section 3 describes the proposed cluster based hybrid scheduling algorithm. Section 4 presents the performance evaluation of grid scheduling algorithms. Conclusion is given in the final section.

\section{Related Research}

A Grid is a high performance computational system which consists of a large number of distributed and heterogeneous resources. Grid computing enables sharing, selection and aggregation of resources to solve the complex large scale problems in science, engineering and commerce. Scientific applications usually consist of numerous jobs that process and generate large datasets. Processing complex scientific applications in a Grid imposes many challenges due to the large number of jobs, file transfers and the storage needed to process them. The scheduling of jobs focuses on mapping and managing the execution of tasks on shared resources [6]. Most of the parallel jobs demand a fixed number of processors, which cannot be changed during execution [7]. Good job scheduling policies are very essential to manage Grid systems in a more efficient and productive way [8]. Grid job scheduling policies can be generally divided into space-sharing and time-sharing approaches. In timesharing policies, processors are temporally shared by jobs. In spacesharing policies, however, processors are exclusively allocated to a single job until its completion. The wellknown space-sharing policies are FCFS, Shortest Job First (SJF), Shortest Remaining Time First (SRTF) and Longest Job First (LJF) approaches. The famous time- sharing scheduling policies are Round Robin (RR) and Proportional Local Round Robin Scheduling (PLRR) [9, 10, and 11]. In [9] the authors have extended the working of basic space sharing techniques like FCFS, SJF, and LJF and proposed an SJF-backfilled scheduling heuristic. First-Come, First-Served Scheduling Algorithm (FCFS) is the simplest algorithm for job scheduling. Jobs are executed according to the sequence of job submitting. The second job will be executed when the first job is done, and therefore FCFS has a serious problem called convoy effect [12]. Or The FCFS is the simplest and non-preemptive job scheduling algorithm. For this algorithm the ready queue is maintained as a FIFO queue. Each new job/process is added to the tail of the ready queue and then the algorithm dispatches processes from the head of the ready queue for execution by the CPU. A process terminates and is deleted from the system after completing its task. The next process is then selected from the head of the ready queue $[10,11]$.

[20] Proposes Grid level resource scheduling with a Job Grouping strategy in order to maximize the resource utilization and minimize the processing time of jobs. A combination of the Best Fit and RR scheduling policies is applied at the local level to achieve better performance. With RR, a fixed time quantum is given to each process that is present in the circular queue, for fair distribution of CPU times. The RR scheduling policy is extensively used for job scheduling in Grid computing [20, 21, and 22].

This paper presents a dynamic cluster based job scheduling algorith $m$ for efficient execution of user jobs. This paper also includes the comparative performance analys is of our proposed job scheduling algorithm along with other well-known job scheduling algorithms considering the parameters like average waiting time, average turnaround time, average response time and average total completion time. The result has shown also exhibit that Our proposed scheduling algorithms (CHS) has shown the best average waiting times, average turnaround times, average response times and average total completion times compared to other job scheduling approaches.

\section{Proposed Cluster based Hybrid Scheduling Algorithm}

In [13, 14 and 15] Shah et al proposed two scheduling algorithms- MH and MDQ. They are based on a fixed time quantum.

In this paper we propose new dynamic cluster based hybrid job scheduling algorithm namely CHS will now be described.

\subsection{Cluster based Hybrid job Scheduling Algorithm (CHS)}


In this method (Fig. 1), a central node considered as Master Node plays the role of taking jobs from users and storing them in its local memory. Then, the jobs are ordered based on CPU calculating burst time, and the jobs are distributed between the clusters. Thereafter, the jobs are numbered from 1 to $\mathrm{n}$ for each cluster. Then, the time quantum of Cluster 1 to Cluster $n$ is calculated in parallel. Then, the cluster with a lower quantum time is CPU allocated and executed.

\subsection{Method of Time Quantum Calculation}

According to the below relation, time quantum is calculated based on the square of CPU burst time average for Cluster 1 to Cluster $\mathrm{n}$ in parallel. Then, the cluster with a lower time quantum compared to other clusters is CPU allocated and executed.
Quantum cluster $1=$
SQRT (Average $(J C T 1, J C T 2, J C T 3, \ldots, J C T i))$

Quantum cluster $2=$ SQRT (Average $(J C T i+1, J C T i+2, J C T i+3, \ldots, J C T j))$

Quantum cluster3 =

SQRT (Average $(J C T j+1, J C T j+2, J C T j+3, \ldots, J C T n))$

In the above relation, the JCT variable represents job processing time. In this proposed method, due to the fact that we cluster the jobs and that time quantum is calculated in parallel for all clusters, hence, this method results in improved waiting time, return time, response time, and total completion time, and acts better than FCFS, RR, LJF, PLRR, and MDQ scheduling algorithms. JCT (Job CPU TIME)

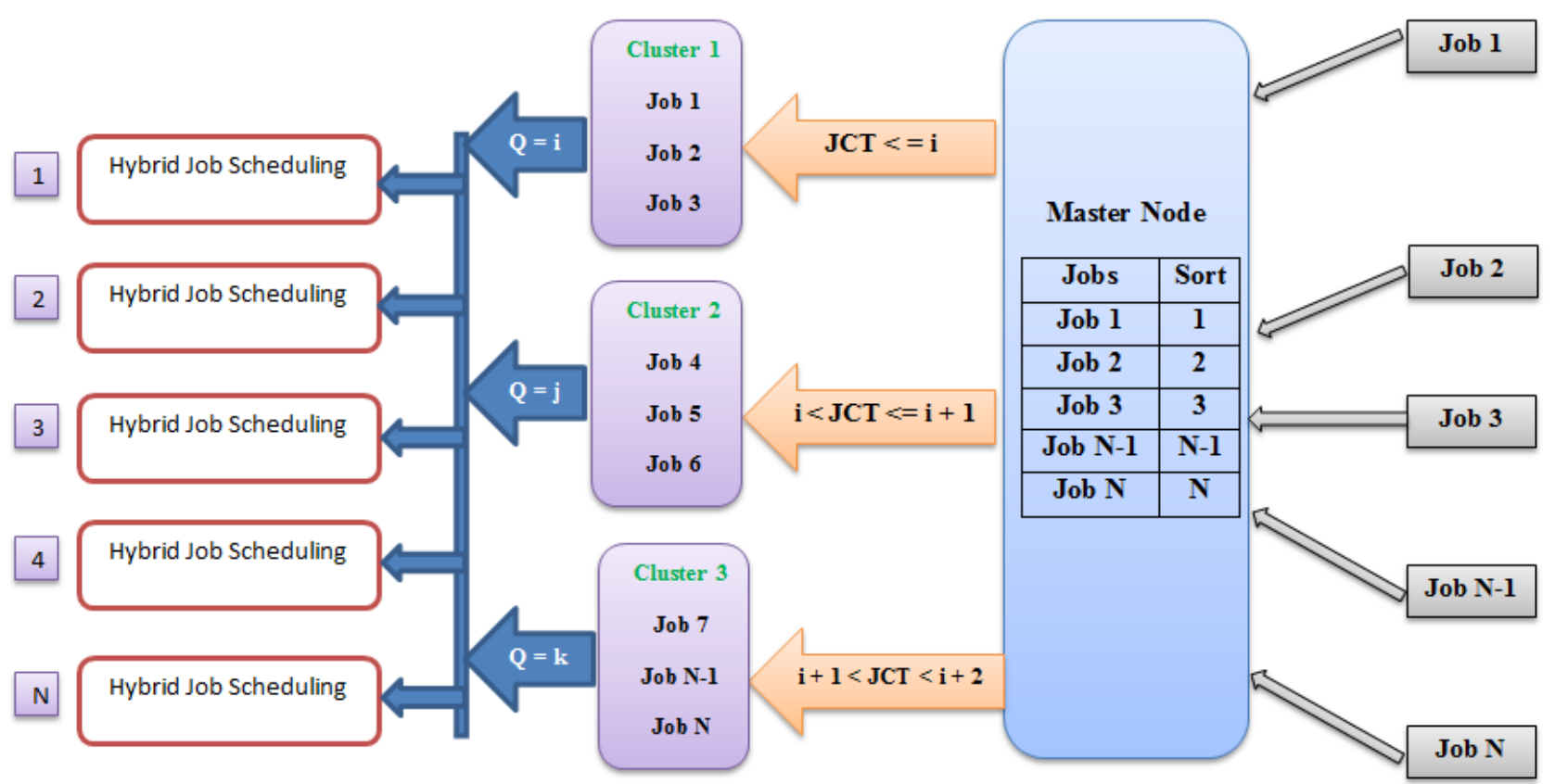

Fig. 1: Block Diagram of Cluster based Hybrid Scheduling (CHS)

\section{Performance Evaluation of Grid Scheduling Algorithms}

Performance metrics for the Grid scheduling algorith ms are based on three factors - Average Waiting Time, Average Turnaround Time, and Average Response Time. We performed experiments for different scheduling algorithms [18]. We formed two data sets by using workload i.e. 19000 and 38000 processes. We performed an experiment by varying the number of CPUs from 8 to 128 . We used '50' units as the fixed time quantum for our experiment. In this section, we describe a comparative performance analys is of our proposed algorithms, i.e. CHS, with six other Grid scheduling algorithms; i.e. FCFS, LJF, SPN, RR, PLRR and MDQ.

\subsection{Average Waiting Times Evaluation}

The Waiting Time is the time for which a process waits from its submission to completion in the local and global queues [16], [17]. Fig. 1 and Fig. 2 shows that the average waiting times computed by each scheduling algorithm for each real workload trace of 19000 and 38000 processes. That the PLRR and CHS scheduling algorithms produce the shortest average waiting times as compared to the other scheduling algorithms. By increasing the number of CPUs, each algorithm shows the relative improvement in performance, except for the FCFS and MDQ algorithms. Also, the FCFS and LJF have shown the worst performance the average waiting time measures. As a result, $\mathrm{CHS}$ has shown the optimal average waiting times for 19000 and 38000 processes. 


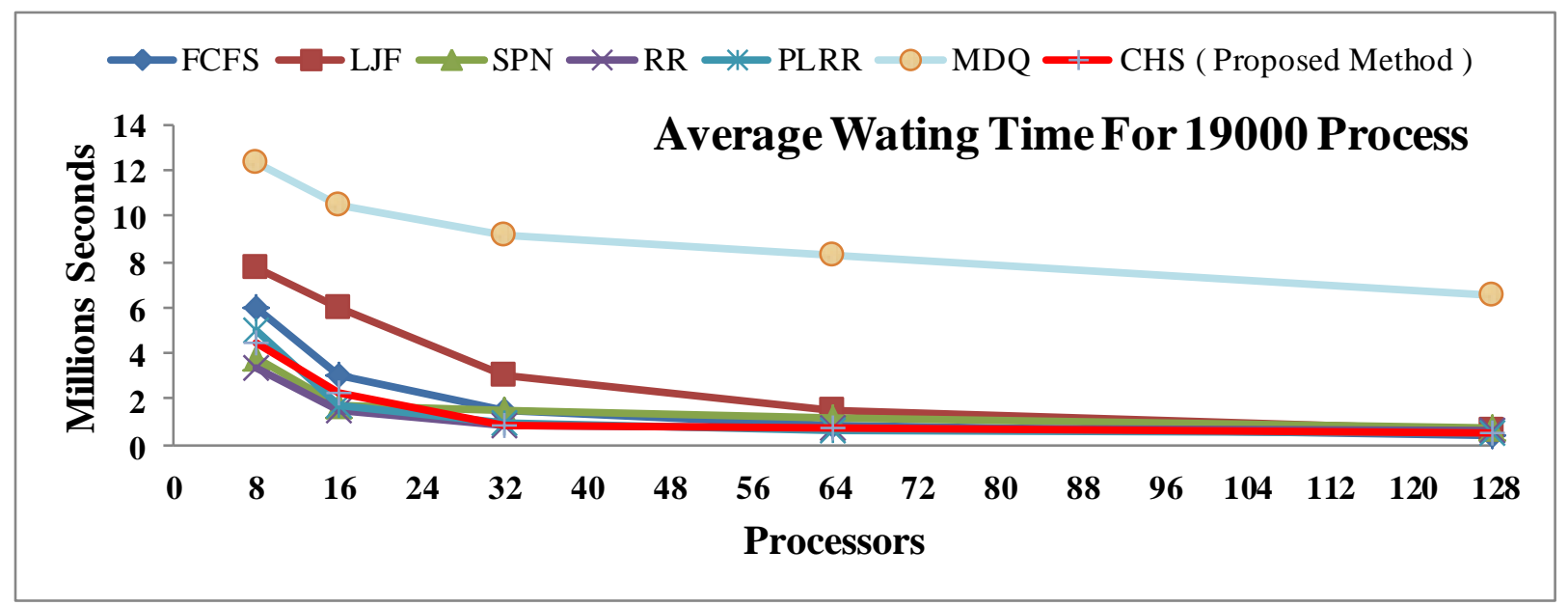

Fig. 1: Average Waiting Time Analysis for 19000 Processes

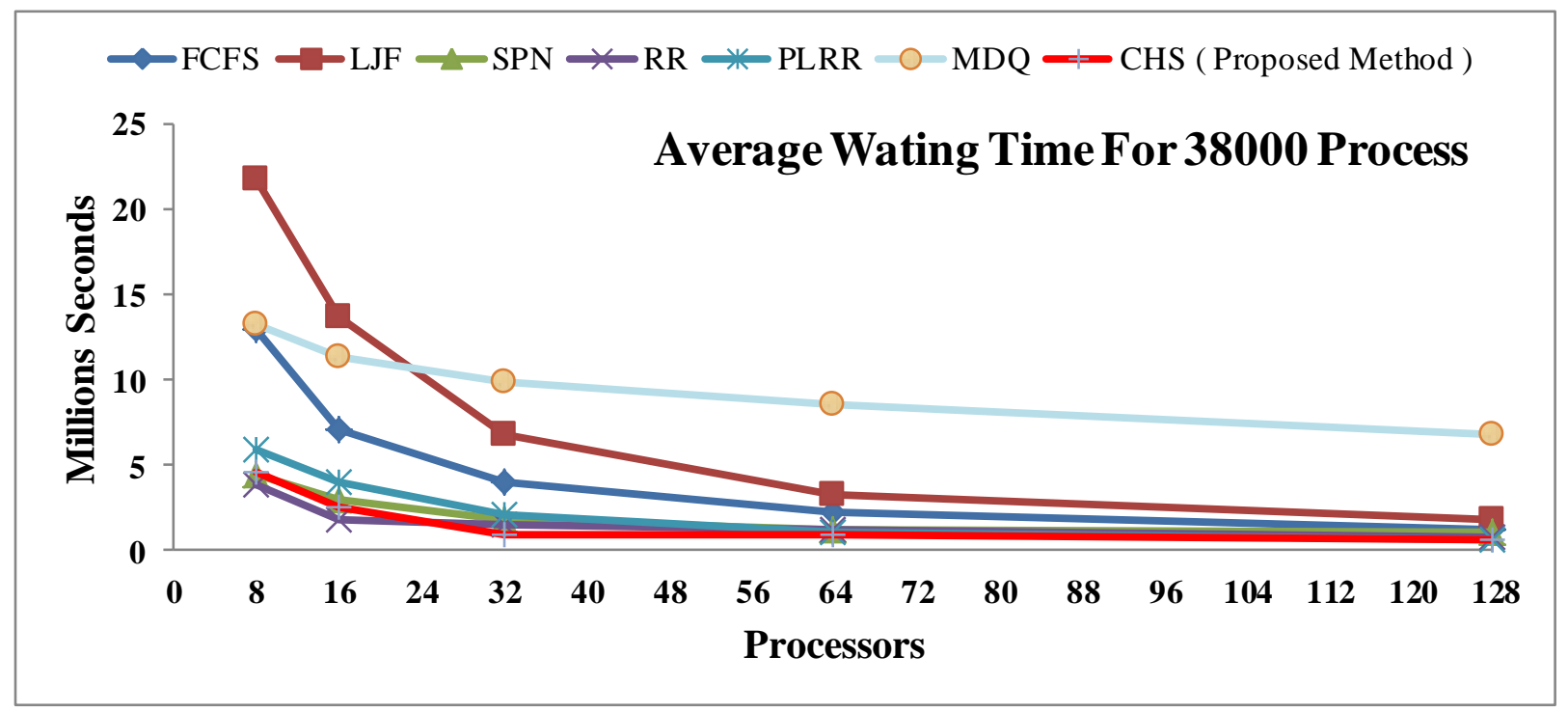

Fig. 2: Average Waiting Time Analysis for 38000 Processes

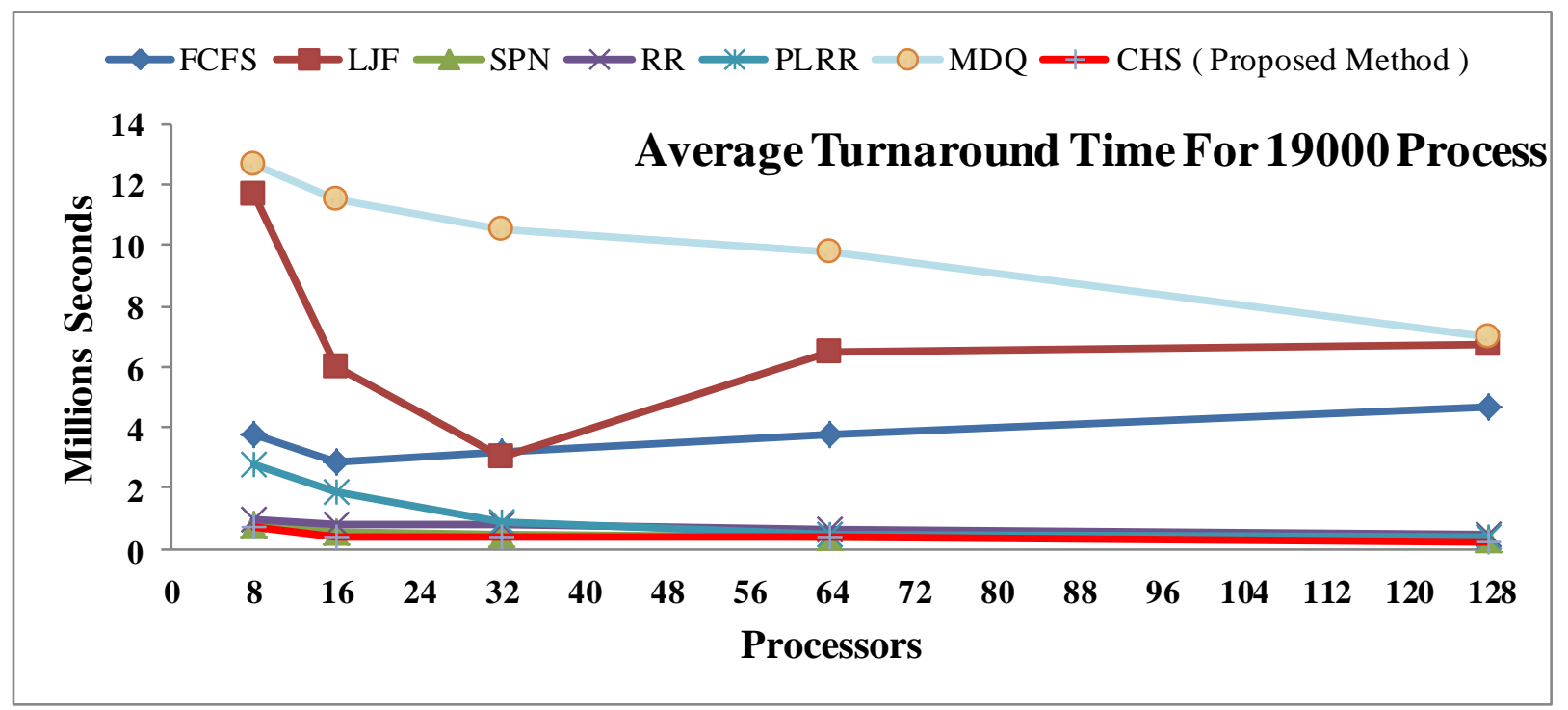

Fig. 3: Average Turnaround Time Analysis for 19000 Processes 


\subsection{Average Turnaround Times Evaluation}

The Turnaround time of the job is defined as the time difference between the completion time and release time [16], [17]. Fig. 3 and Fig. 4 shows that the average turnaround times computed by each scheduling algorithm for each real workload trace of 19000 and 38000 processes, That the average turnaround time computed by the RR, PLRR and CHS scheduling algorithms are shorter than the other Grid scheduling algorithms, Also By increasing the number of CPUs, each algorithm has an improved average turnaround time, except for the LJF and FCFS scheduling algorithm. Furthermore, it is found that FCFS, MDQ and LJF scheduling algorithms have shown the longer average turnaround time measures.

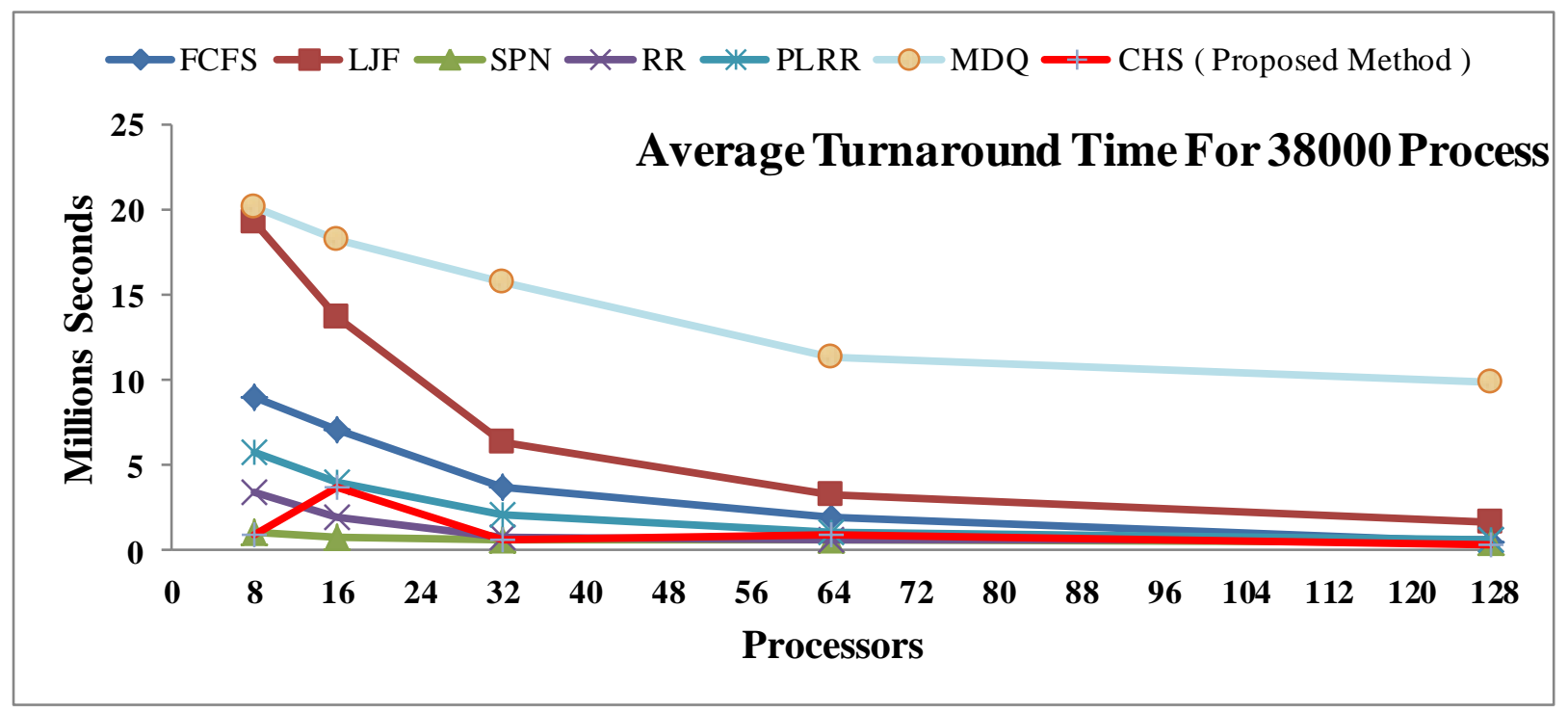

Fig. 4: Average Turnaround Time Analysis for 38000 Processes

\subsection{Average Response Times Evaluation}

It is the amount of time taken from when a process is submitted until the first response is produced [16], [17]. Average response times for each algorithm have decreased by increasing the number of CPUs. Fig. 5 and Fig. 6 shows that the average response times computed by each scheduling algorithm for each real workload trace of 19000 and 38000 processes, The SPN, LJF and
FCFS scheduling algorithms result in poor response times as compared to the other scheduling algorith ms. It also shows that MDQ and RR algorithms produces better average response time compared to other algorith ms. However, FCFS, PLRR, SPN and LJF have shown the worst performance average response time measures, out of which LJF results in the longest average response times.

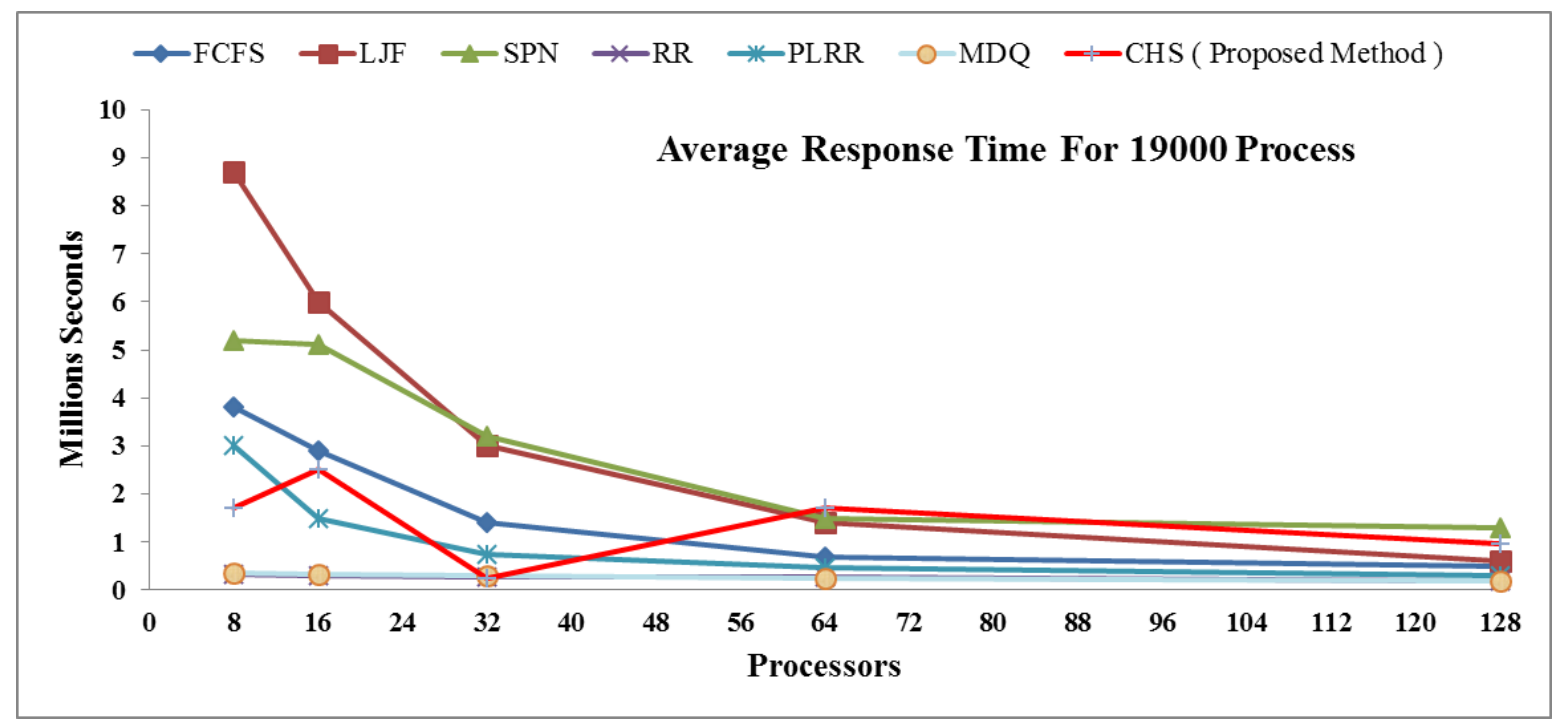

Fig. 5: Average Response Time Analysis for 19000 Processes 


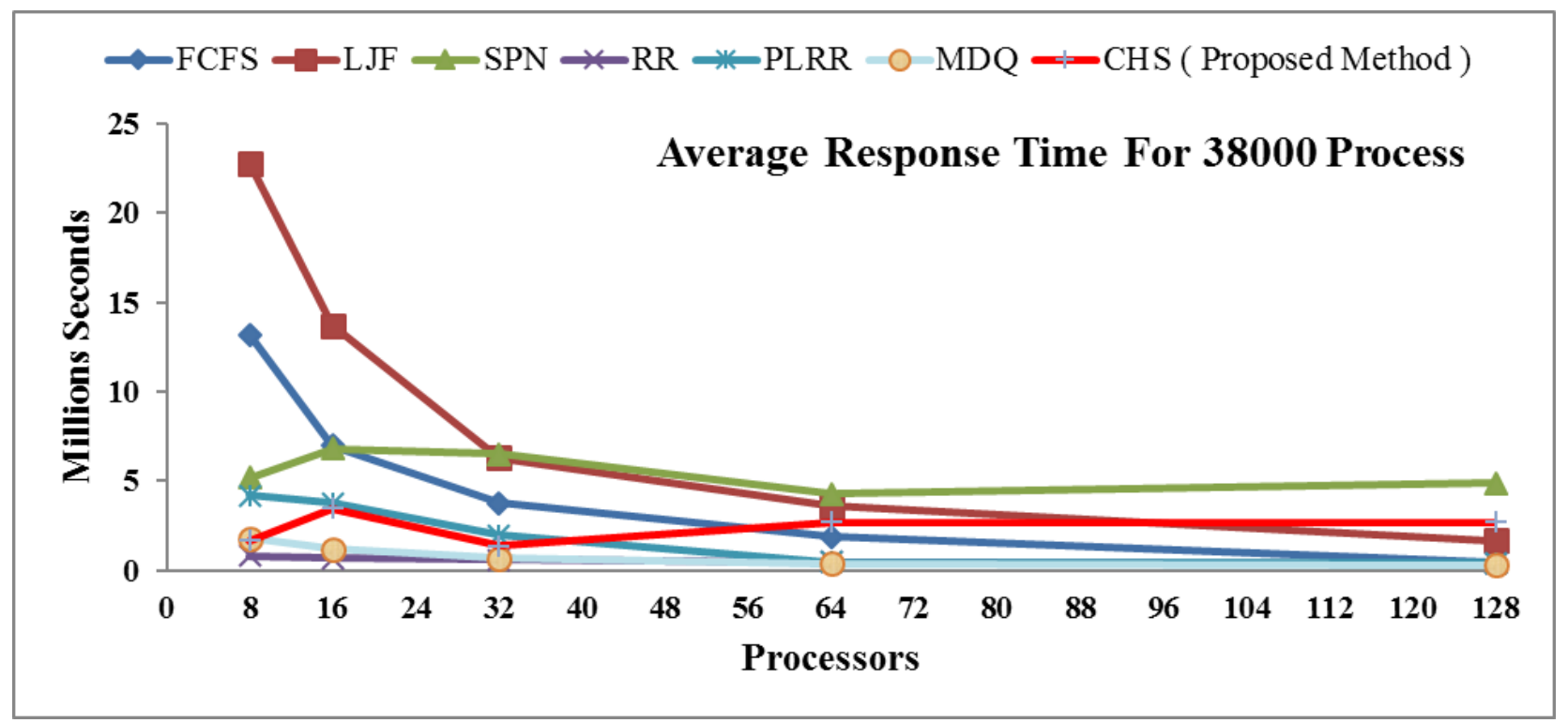

Fig. 6: Average Response Time Analysis for 38000 Processes

\subsection{Average Total Completion Times Evaluation}

Machine Completion time is defined as the time for which a machine ' $m$ ' will finalize the processing of the previously assigned tasks as well as of those already planned tasks for the machine [19]. Fig. 7 and Fig. 8 shows that the average total completion times computed by each scheduling algorithm for each real workload trace of 19000 and 38000 processes, That the average total completion times computed by the RR, SPN and CHS scheduling algorithms are shorter than the other Grid scheduling algorithms, it is found that FCFS, MDQ, PLRR and LJF scheduling algorithms have shown the longer average turnaround time measures.

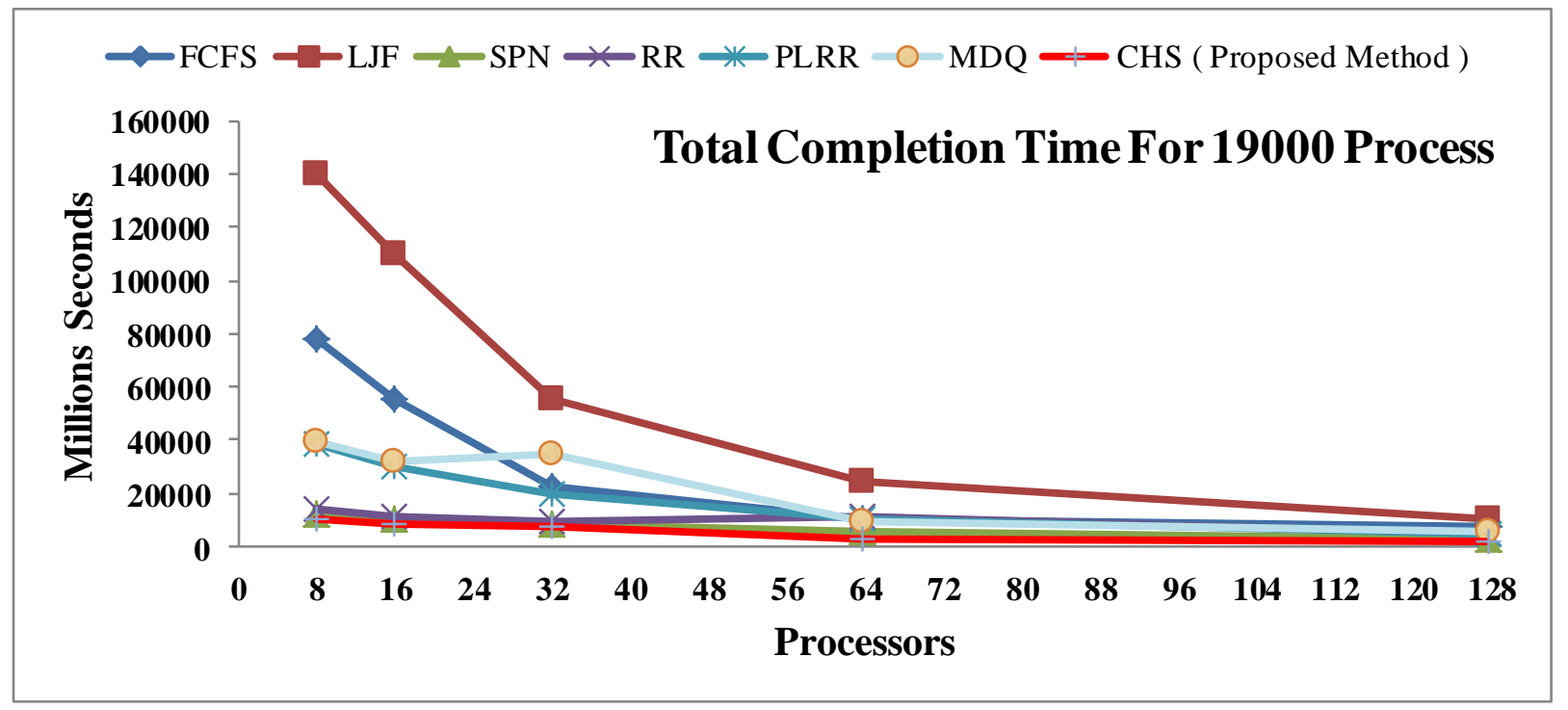

Fig. 7: Average Total Completion Time Analysis for 19000 Processes 


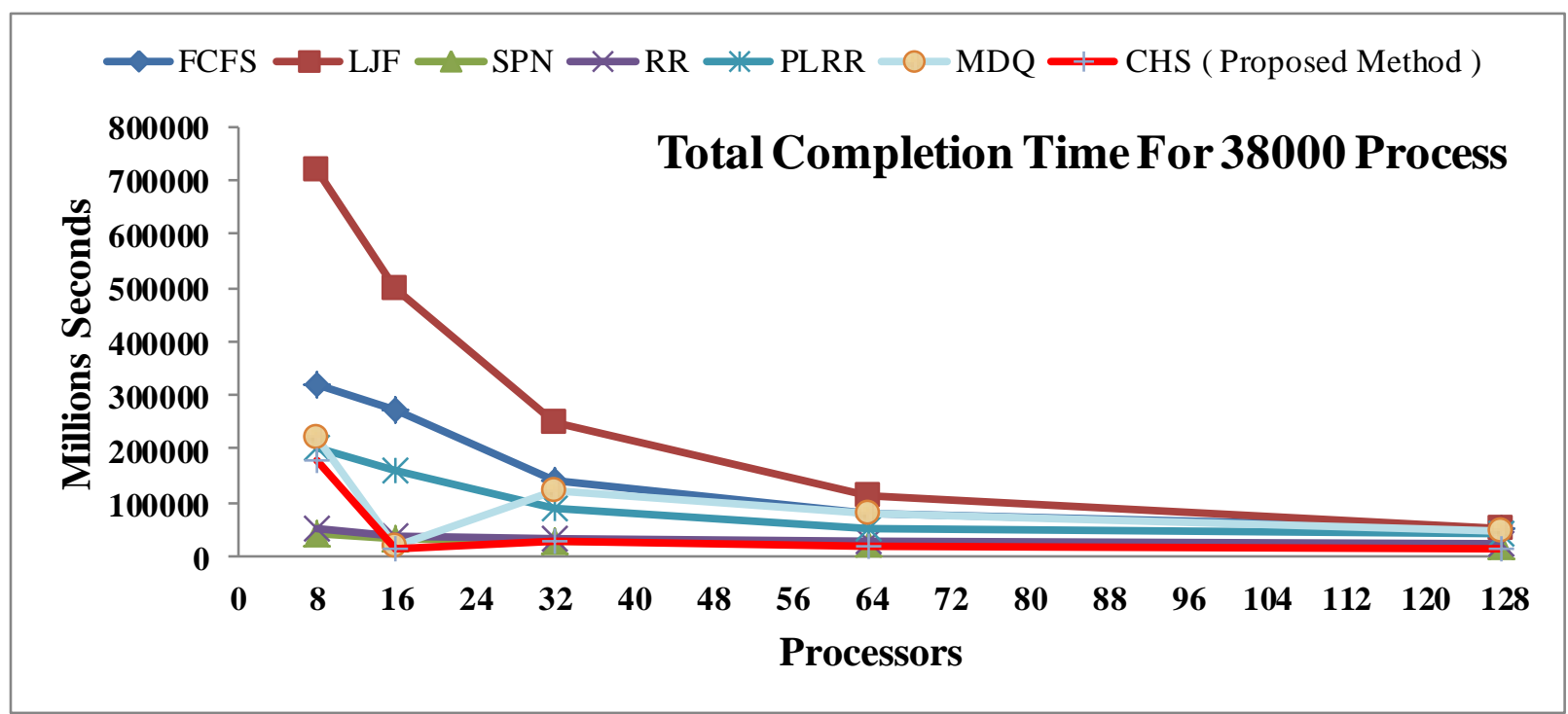

Fig. 8: Average Total Completion Time Analysis for 38000 Processes

\section{Conclusion}

In this paper, the architecture of a cluster-based scheduling framework of the Grid computing is proposed, namely CHS. We compared the performance of proposed job scheduling algorithm with other grid scheduling algorithms on a computational grid. Simulation results show that CHS has shown the optimal performance in terms of average waiting times, average turnaround times, and total completion times. Simulation results also exhibit that MDQ and RR has shown the best average response times compared to other job scheduling approaches.

\section{References}

[1] E. Shmueli and D. G. Feitelson, "Backfilling with lookahead to optimize the packing of parallel jobs," J. Parallel Distrib. Comput., vol. 65, pp. 1090-1107, 2005.

[2] Y. Zhang, H. Franke, J. E. Moreira, and A. Sivasubramaniam, "Improving parallel job scheduling by combining gang scheduling and backfilling techniques," in Parallel and Distributed Processing Symposium, 2000. IPDPS 2000. Proceedings. 14th International, 2000, pp. 133-142.

[3] SETI@home, http://setiathmoe.berkeley.edu/>.

[4] The Data TransAtlantic Grid Project, $<$ http://datatag.web.cern.ch/datatag/>.

[5] Globus Toolkit, <http://www.globus.org/toolkit/>.

[6] I. Foster and C. Kesselman, the Grid: Blueprint for a New Computing Infrastructure, Morgan Kaufmann, 1999.

[7] E. Shmueli and D.G. Feitelson, Backfilling with look ahead to optimize the packing of parallel jobs.
J Parallel Distrib Comput, vol. 65, no. 9, pp. 1090 1107. ISSN 0743-7315, 2005

[8] Y. Zhang, H. Franke and J.E. Moreira, A. Sivasubramaniam, Improving parallel job scheduling by combining gang scheduling and Backfilling techniques. In: Parallel and distributed processing symposium, IPDPS 2002, pp. 133-142. ISBN: 0-7695-0574-0, 2002.

[9] B. Lawson, E. Smirni and D. Puiu, Self-adaptive backfill scheduling for parallel systems. In: Proceedings of the international on ference on parallel processing (ICPP 2002), pp. 583-592, and 2002

[10] D. Tsafrir, Y. Etsion and D. G. Feitelson, Backfilling using system-generated predictions rather than user runtime estimates. IEEE Trans Parallel Distrib Syst, vol. 18, no. 6, pp. 789-803. ISSN: 1045-9219, 2007

[11] J.H. Abawajy, Job Scheduling Policy for High Throughput Grid Computing, Lecture Notes in Computer Science, Springer, 2005

[12] Abraham Silberschatz, Peter Baer Galvin, Greg Gagne, Operating System Concepts, eighth ed., John Wiley \& Sons, 2011.

[13] S. N. M. Shah, A. K. B. Mahmood and A. Oxley, Development and Performance Analysis of Grid Scheduling Algorithms, Communications in Computer and Information Science, Springer, vol. 55, pp. 170-181, 2009

[14] S. N. M. Shah, A. K. B. Mahmood and A. Oxley, Hybrid Scheduling and Dual Queue Scheduling, 2009 the 2nd IEEE International Conference on Computer Science and Information Technology (IEEE ICCSIT 2009), 8-11 Aug 2009

[15] S. N. M. Shah, A. K. B. Mahmood and A. Oxley, 
Analysis and Evaluation of Grid Scheduling Algorithms using Real Workload Traces, The International ACM Conference on Management of Emergent Digital EcoSystems, MEDES'10, 26-29 October 2010.

[16] W. Stallings, Operating Systems Internals and Design Principles: Prentice Hall, 2004.

[17] J. Blazewicz, Ecker, K.H., Pesch, E., Schmidt, G. und J. Weglarz, Scheduling Computer and Manufacturing Processes:Berlin (Springer), 2001.

[18] S. N. M. Shah, A. K. B. Mahmood and A. Oxley, Dynamic Hybrid Scheduling Algorithms for Grid Computing, 2011 International Conference on Computer Science (Science Direct ICCS 2011), 402-411.

[19] F. Xhafa and A. Abraham, "Computational models and heuristic methods for Grid scheduling problems," Future Gener. Comput. Syst., vol. 26, pp. 608-621, 2010.

[20] R. Sharma, V. K. Soni and M. K. Mishra, An Improved Resource Scheduling Approach Using Job Grouping strategy in Grid Computing, 2010 International Cornference on Educational and Network Technology, 2010

[21] J.H. Abawajy, Job Scheduling Policy for High Throughput Grid Computing, Lecture Notes in Computer Science, Springer, 2005

[22] T Laurence, M. G. Yang, Chapter 17 of "HighPerformance Computing: Paradigm and Infrastructure", Wiley, ISBN: 978-0-471-65471-1, 2005

\section{Authors' Profiles}

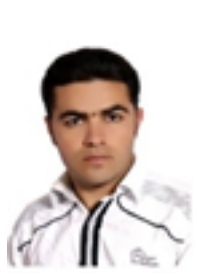

Reza Fotohi received his B.Sc. in computer engineering from Shabestar University of Applied Science And Technology, Tabriz, Iran, in 2009, and his M.Sc. in Computer Engineering from Islamic Azad University, Germi branch, Ardabil, Iran, in 2013. His research interests include Mobile Ad-hoc Networks, Performance Evaluation, and Optimization Algorithms.

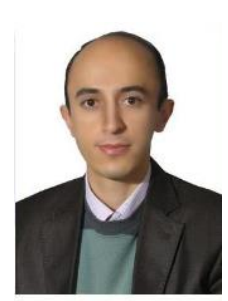

Mehdi Effatparvar is faculty member of computer engineering department in Islamic Azad University of Ardabil, Iran. He is PhD student in Islamic Azad University of Science and Research. He received his BSc in Computer engineering and MSc in Information Technology from Islamic Azad University of Qazvin, Iran. His research interests include wireless sensor networks, ad-hoc networks, distributed systems and operating systems.
How to cite this paper: Reza Fotohi, Mehdi Effatparvar,"A Cluster Based Job Schedu ling Algorithm for Grid Computing", International Journal of Information Technology and Computer Science(IJITCS), vol.5, no.12, pp.70-77, 2013. DOI: 10.5815/ijitcs.2013.12.09 